\title{
ANÁLISIS DE FACTORES ASOCIADOS A FLUOROSIS DENTAL MEDIANTE APLICACIÓN DE LA FICHA DE NOTIFICACIÓN DE EXPOSICIÓN A FLÚOR EN LAS CLÍNICAS ODONTOLÓGICAS DE LA UNIVERSIDAD SANTO TOMÁS, 2014
}

\author{
${ }^{1}$ Olga Viviana Cañas Vera, ${ }^{1}$ Andrea Carolina Velásquez Torres, ${ }^{1}$ Geyme Paola Zapata Rey, ${ }^{2}$ Francisco Tirado Santamaría \\ ${ }^{1}$ Estudiante de X semestre de Odontología de la U. Santo Tomás, Bucaramanga, ${ }^{2} E s p$. en Epidemiologia, Universidad CES, Docente U. Industrial de Santander
}

Autora responsable de correspondencia: Geyme Paola Zapata Rey

Correo electrónico: gepazare@hotmail.com

\begin{abstract}
RESUMEN
Objetivo: describir los factores asociados a fluorosis dental en los pacientes que asisten a consulta en las Clínicas Odontológicas de la Universidad Santo Tomás, mediante la ficha de notificación obligatoria de exposición al flúor en la USTA 2014.

Materiales y métodos: Se llevó a cabo un estudio observacional, analítico de corte transversal. la población estuvo conformada por 144 niños entre los 6 a 12 años que acudieron a consulta odontológica y presentaron fluorosis dental, se calculó el tamaño de muestra con el programa Epi-Info versión 3.2.2.

Resultados: el promedio de edad de los participantes fue de 8,2 años, el estrato socioeconómico más común fue 2 (40,3\%), la clasificación de fluorosis más frecuente fue leve $(51,4 \%)$, se detectó presencia de caries en la mayoría de los pacientes $(85,4 \%)$. Los niños fueron los responsables del cepillado en el $66 \%$ de los casos, es habitual que se cepillen 2 veces al día $(52,1 \%)$, que la cantidad de crema que más usen sea $3 / 3$ de la cabeza del cepillo (50\%) y la ingesta de crema dental durante el cepillado fue del 38,2\%.

Conclusiones: factores relacionados con el uso inadecuado de cremas dentales siguen jugando un rol importante en la incidencia de fluorosis dental, el nivel socioeconómico es un factor trascendental en cuanto a las condiciones de salud y hábitos de higiene y cuidado bucal. Se detecta la necesidad de mejorar la educación en cuanto a los hábitos adecuados de higiene oral, como frecuencia de cepillado, cantidad de crema utilizada y acompañamiento oportuno en los primeros años de vida del niño, para evitar alteraciones como la fluorosis. [Cañas O, Velásquez A, Zapata G, Tirado F. Análisis de factores asociados a fluorosis dental mediante aplicación de la ficha de notificación de exposición a flúor en las clínicas odontológicas de la USTA 2014. Ustasalud. 2014;13(2): 144 - 150]
\end{abstract}

Palabras clave: Fluorosis dental, Caries dental, Flúor.

\section{ANALYSIS OF FACTORS ASSOCIATED WITH DENTAL FLUOROSIS BY APPLICATION OF FLUORIDE EXPOSURE NOTIFICATION FORM IN DENTAL CLINICS AT UNIVERSIDAD SANTO TOMÁS-2014}

\begin{abstract}
Objective: to describe the basic clinical characteristics and the level of dental fluorosis in the patients of the dental clinics of the Universidad Santo Tomás through the record notifiable exposure to fluoride in the USTA 2014.

Methods: the population consisted of 144 children between the ages of 6 and 12, who attend dental consultations and have dental fluorosis, the sample size was calculated using the Epi-Info, version 3.2.2 program. A cross sectional study was conducted.

Results: the mean age of the participants was 8.2 years, the most common socioeconomic stratum was 2 (40.3\%), the most frequent fluorosis classification was mild (51.4\%), presence of caries was detected in the Majority of patients (85.4\%). The children were responsible for brushing in $66 \%$ of cases, it is common to brush twice a day $(52.1 \%)$, that the amount of cream they use most is $3 / 3$ of the head of the brush (50\%) And toothpaste intake during brushing was $38.2 \%$.

Conclusions: factors related to the inadequate use of toothpaste continue to play an important role in the incidence of dental fluorosis, socioeconomic status is a major factor in terms of health and hygiene habits and oral care. The need to improve education regarding adequate oral hygiene habits such as brushing frequency, amount of cream to be used and timely accompaniment in the first years of the child's life is detected, to avoid alterations such as fluorosis.
\end{abstract}

Key words: Dental fluorosis, Dental caries, Fluoride.

Recibido para publicación: 10 de octubre de 2014. Aprobado para publicación: 23 de noviembre de 2014. 


\section{INTRODUCCIÓN}

El uso de flúor (F) en cremas dentales, suplementos, sal, leche, entre otros, al igual que la fluoración de los suministros de agua, han jugado un papel importante en la prevención de la caries dental, debido a que se consideran un método eficaz, seguro y necesario para combatirla. Sin embargo, numerosos estudios han demostrado resultados adversos causados por el efecto acumulativo de este elemento a través de los años, lo que indica que la exposición total al flúor ha aumentado al ser suministrado por diferentes fuentes de manera simultánea, causando diversas alteraciones, no solo bucales como la fluorosis dental, sino además afectando distintos sistemas del organismo ${ }^{1}$.

El flúor no es un elemento esencial para la vida, pero constituye una alternativa preventiva muy versátil, a través de los hallazgos y el análisis de su comportamiento se ha identificado que puede producir efectos benéficos o adversos para la salud según sea su uso. En la dentición, contribuye a aumentar la resistencia de los tejidos dentales, incrementando el grado de remineralización del esmalte, reduciendo la solubilidad del tejido que ha estado expuesto a un medio ácido ${ }^{2}$.

Con respecto al comportamiento del evento a nivel mundial, en las últimas décadas se ha observado un aumento de la prevalencia de fluorosis dental, con porcentajes que van de $7,7 \%$ a $80,7 \%$ en áreas donde se cuenta con agua fluorada y entre $2,9 \%$ a $42 \%$ en áreas sin agua fluorada ${ }^{3-6}$.

Un factor de riesgo para la fluorosis dental es el alto contenido de flúor en agua y algunos municipios de Santander se han caracterizado por presentar dicha situación, ya que las fuentes de agua registran niveles elevados de flúor (0,5 ppm); en estudios realizados en el 2003 por Concha S. y cols. los casos de fluorosis encontrados en Bucaramanga (Santander) fueron del $77,7 \%$, niveles muy altos comparados con otros departamentos del país ${ }^{7-11}$.

Aunque hay pocos datos poblacionales de vigilancia epidemiológica que demuestren los efectos ocasionados tras la introducción de la fluoración de la sal y exposición a otras fuentes de fluoruros en Colombia $^{12}$, investigaciones han demostrado que los casos de fluorosis siguen siendo una alarma en el país ${ }^{11}$, por lo que se considera necesario e importante realizar estudios que permitan conocer el comportamiento de esta alteración en la comunidad.

La Universidad Santo Tomás como institución prestadora de servicios de salud (IPS), al seguir aplicando la ficha de notificación para el registro de los casos de fluorosis que se presentan en la consulta, con- tará con mayor información acerca de los factores que pudieron desencadenar la alteración, además de esto los pacientes requieren ser diagnosticados correctamente, de esta manera podrá realizarse un tratamiento adecuado y conocer qué factores pudieron desencadenar la patología para prevenirla en los demás miembros de la familia o comunidad, en especial en los niños; velando así por la protección de la salud individual y colectiva, con el fin de mejorar los indicadores de salud bucal como aporte a la calidad de vida de las personas ${ }^{11-14}$.

Por lo tanto, el objetivo del presente trabajo fue describir factores asociados a la fluorosis dental mediante los resultados obtenidos con la aplicación de la ficha de notificación obligatoria de la exposición al flúor en las clínicas odontológicas de la Universidad Santo Tomás.

\section{MATERIALES Y MÉTODOS}

Se realizó un estudio de corte transversal y se aplicó la ficha de notificación obligatoria de exposición al flúor en las Clínicas Odontológicas de la Universidad Santo Tomás, durante el II período de 2014. El cálculo del tamaño de muestra se realizó en el programa Epi-Info versión 3.2.2, partiendo de una frecuencia esperada del $52,8 \%{ }^{10}$ con un $95 \%$ de confianza y un error tipo I de 5\%, con un total población de 196 niños que consultaron, la muestra estuvo conformada por 144 niños que cumplieron con los criterios de inclusión.

Se llevó a cabo un muestreo no probabilístico por conveniencia de los niños con fluorosis dental que consultaron en las Clínicas Odontológicas. Como criterios de inclusión se tuvieron en cuenta todos los niños que acudieron a consulta a las Clínicas Odontológicas de la Universidad Santo Tomás, que estuvieran en un rango de edad de 6 a 12 años, dichos participantes debían presentar algún tipo de fluorosis dental, tener una conducta que fuera apta para el examen, además de no presentar ningún síndrome o discapacidad que dificultara la valoración clínica. No se incluyeron niños cuyos padres o tutores no autorizaran la participación en el estudio, pacientes con dentición temporal completa, es decir, que no tengan en boca ningún diente permanente, ni con antecedentes de ingesta de la madre de tetraciclinas durante la gestación o primera infancia.

El método que se utilizó para la recolección de la información fue la ficha de notificación obligatoria de exposición a flúor del Sistema Nacional de Vigilancia en Salud Pública (SIVIGILA), del Instituto Nacional de Salud.

La selección de los participantes se realizó durante la atención a pacientes en las clínicas de Pediatría 
I, II y III, se detectaron los niños que se encontraban en consulta y se diligenció la ficha solo a los que presentaban fluorosis dental, hasta completar el tamaño de la muestra; previamente se entregó al padre de familia o responsable del niño un consentimiento informado, mediante el que se les explicó claramente el objetivo del estudio y los procedimientos a llevarse a cabo.

El análisis de los datos obtenidos se procesó con el programa Epi-Info versión 3.2.2 de 2004, en el subprograma análisis de datos se calcularon medidas de tendencia central y dispersión para las variables cuantitativas y distribución de frecuencia para las variables cualitativas; el análisis bivariado se hizo mediante la prueba de $\mathrm{Chi}^{2}$, con una confianza del $95 \%$.

\section{Consideraciones éticas}

Según la Resolución 008430 de octubre de 1993, emitida por el Ministerio de Salud, en la cual se establecen las normas científicas, técnicas y administrativas para investigación en salud, se consideró que esta fue una investigación de riesgo mínimo, ya que se recolectaron datos a través de procedimientos comunes, consistentes en examen clínico y se aplicó un cuestionario. A todos los participantes y sus acompañantes se les socializaron los detalles del estudio y firmaron el consentimiento informado.

\section{RESULTADOS}

Según las variables sociodemográficas la población estuvo integrada por 144 niños de género masculino y femenino. El promedio de edad fue 8,2 años con un intervalo de edad entre 6 a 12 años, la edad más frecuente 9 años con 22,9\% (33), seguido de 8 y 6 años con 20,1\% (29) cada uno. En el grado de escolaridad el mayor porcentaje se presentó en la primaria con 94,4\% (136), El lugar de residencia habitual de los niños los primeros 9 años de vida fue la zona urbana con $93,8 \%$ (135). En lo que concierne al estrato socioeconómico, el más común fue el estrato 2 con 40,3\% (58) (Tabla 1).

En relación con las características clínicas dentales de los pacientes observados, en la clasificación de las lesiones (Índice de DEAN) de clasificación del paciente, la de mayor frecuencia fue leve con $51,4 \%$ (74), seguido de moderada con $26,4 \%$ (38) y muy leve con $16,7 \%$ (24). Se encontró presencia de caries en la mayoría de los pacientes examinados con un $85,4 \%$ (123) y el tipo de caries que más se halló fue cavitacional con 79,9\% (115) (Tabla 2).

Acerca de los hábitos y parámetros de higiene oral se encontró que el responsable del cepillado dental, más reiterado, fue el mismo niño menor de 8 años, con $66 \%$ (95), la frecuencia de cepillado con crema dental en los niños observados lo más común fue dos veces al día con $52,1 \%$ (75), la cantidad de crema dental que más usan es $3 / 3$ de la cabeza del cepillo con $50 \%$ (72), se registra ingesta de crema dental durante el cepillado en el 38,2\% (55) y no hubo aplicaciones tópicas de flúor en el 65,3\% (94) de los casos (Tabla 3).

Según el análisis bivariado con respecto a las variables socioeconómicas (estrato) y su influencia en la presencia y severidad de alteraciones bucales tales como fluorosis y caries dental, no se presentó diferencia estadísticamente significativa en relación con el estrato y la severidad de la fluorosis dental $(p=0,610)$, pero si se encontró asociación estadísticamente significativa entre el estrato y la presencia de caries dental $(p=0,050)$ (Tabla 4$)$.

Tabla 1. Distribución de características sociodemográficas de pacientes que se les diligenció la ficha de notificación de fluorosis en las Clínicas Odontológicas Universidad Santo Tomás, 2014

\begin{tabular}{lcc}
\hline \multicolumn{1}{c}{ Variables } & $\mathbf{n}$ & $\%$ \\
\hline Edad & 29 & 20,1 \\
6 años & 22 & 15,3 \\
7 años & 29 & 20,1 \\
8 años & 33 & 22,9 \\
9 años & 16 & 11,1 \\
10 años & 8 & 5,6 \\
11 años & 7 & 4,9 \\
12 años & & \\
Escolaridad & 1 & 0,7 \\
Pre escolar & 136 & 94,4 \\
Primaria & 6 & 4,2 \\
Secundaria & 1 & 0,7 \\
Ninguna & & \\
Lugar de residencia & 135 & 93,8 \\
Zona urbana & 8 & 5,6 \\
Zona rural & 1 & 0,7 \\
Otro país & & \\
Estrato socioeconómico & 32 & 22,2 \\
Estrato 1 & 58 & 40,3 \\
Estrato 2 & 40 & 27,8 \\
Estrato 3 & 13 & 9,0 \\
Estrato 4 & 1 & 0,7 \\
Estrato 5 & &
\end{tabular}




\section{ARTÍCULO DE INVESTIGACIÓN CIENTÎFICA Y TECNOLÓGICA}

Tabla 2. Distribución de variables de interés clínico de pacientes que se les diligenció la ficha de notificación de fluorosis en las Clínicas Odontológicas Universidad Santo Tomás, 2014

\begin{tabular}{|c|c|c|c|}
\hline & Variables & $\mathrm{n}$ & $\%$ \\
\hline \multicolumn{4}{|l|}{ Índice de Dean } \\
\hline Dudoso & & 2 & 1,4 \\
\hline Muy leve & & 24 & 16,7 \\
\hline Leve & & 74 & 51,4 \\
\hline Moderada & & 38 & 26,4 \\
\hline Severa & & 6 & 4,2 \\
\hline \multicolumn{4}{|c|}{ Presencia de caries } \\
\hline Sí & & 123 & 85,4 \\
\hline No & & 21 & 14,6 \\
\hline \multicolumn{4}{|l|}{ Tipo de caries } \\
\hline No aplica & & 21 & 14,6 \\
\hline No cavitacional & & 8 & 5,6 \\
\hline Cavitacional & & 115 & 19,9 \\
\hline
\end{tabular}

Tabla 3. Distribución de los hábitos de higiene oral de pacientes que se les diligenció la ficha de notificación de fluorosis en las Clínicas Odontológicas Universidad Santo Tomás, 2014

\begin{tabular}{lcc}
\hline \multicolumn{1}{c}{ Variables } & $\mathbf{n}$ & $\%$ \\
\hline Responsable del cepillado del niño antes de los 8 años & & \\
La madre o el cuidador & 49 & 34 \\
El niño & 95 & 66 \\
Frecuencia de cepillado con crema dental/al día & \\
Una vez & 28 & 19,4 \\
Dos veces & 75 & 52,1 \\
Tres veces & 38 & 26,4 \\
Cuatro veces & 3 & 2,1 \\
Cantidad de crema usada según el tamaño de la cabeza del cepillo dental & \\
$1 / 3$ & 5 & 3,5 \\
$2 / 3$ & 67 & 46,5 \\
$3 / 3$ & 72 & 50 \\
Ingesta de crema dental durante el cepillado & & \\
Sí & 55 & 38,2 \\
No & 89 & 61,8 \\
Ingesta de enjuague bucal & & \\
No & 144 & 100 \\
Aplicación tópica de flúor en el último año & & \\
Sí & 50 & 34,7 \\
No & 94 & 65,3 \\
\hline & & \\
\hline
\end{tabular}


Tabla 4. Influencia del estrato socioeconómico en la presencia y severidad de alteraciones bucales de pacientes que se les diligenció la ficha de notificación de fluorosis en las Clínicas Odontológicas Universidad Santo Tomás, 2014

\begin{tabular}{lcccccc}
\hline \multicolumn{1}{c}{ Variables } & \multicolumn{5}{c}{ Estrato socioeconómico } \\
\hline \multicolumn{1}{c}{$\mathrm{n}(\%)$} & Estrato 1 & Estrato 2 & Estrato 3 & Estrato 4 & Estrato 5 & Valor p \\
\hline & $32(22,2)$ & $58(40,3)$ & $40(27,8)$ & $13(9)$ & $1(0,7)$ & \\
Índice de DEAN & & & & & & 0,061 \\
Dudoso & - & $1(1,7)$ & $1(2,5)$ & - & - & \\
Muy leve & $5(15,6)$ & $10(17,2)$ & $5(12,5)$ & $4(30,8)$ & - & \\
Leve & $14(43,8)$ & $32(55,2)$ & $23(57,5)$ & $4(30,8)$ & $1(100)$ & \\
Moderada & $9(28,1)$ & $14(24,1)$ & $10(25)$ & $5(38,5)$ & - & \\
Severa & $4(12,5)$ & $1(1,7)$ & $1(2,5)$ & - & - & \\
Presencia de caries & & & & & & \\
Sí & $28(22,8)$ & $51(41,5)$ & $35(28,5)$ & $9(7,3)$ & - & \\
No & $4(19)$ & $7(33,3)$ & $5(23,8)$ & $4(19)$ & $1(4,8)$ & \\
\hline
\end{tabular}

Con respecto a otras conductas que pueden ser desencadenantes de fluorosis dental, tales como el encargado o responsable del cepillado del niño cuando fue menor de 8 años y su relación con la cantidad de crema que este usa, se presentó asociación esta- dísticamente significativa $(\mathrm{p}=0,020)$ (Tabla 5). Entre la ingesta de crema dental durante el cepillado y la frecuencia de cepillado no se presentó relación (Tabla 6).

Tabla 5. Distribución de la cantidad de crema, según responsable del cepillado de pacientes que se les diligenció la ficha de notificación de fluorosis en las Clínicas Odontológicas Universidad Santo Tomás, 2014

\begin{tabular}{|c|c|c|c|}
\hline Variables & \multicolumn{3}{|c|}{ Responsable del cepillado cuando el paciente fue menor de 8 años } \\
\hline n (\%) & $\begin{array}{l}\text { Madre } \\
49(34)\end{array}$ & $\begin{array}{l}\text { El niño } \\
95 \text { (66) }\end{array}$ & Valor de $\mathrm{p}$ \\
\hline \multicolumn{4}{|c|}{ Cantidad de crema usada según el tamaño de la cabeza del cepillo dental } \\
\hline $1 / 3$ & $3(6,1)$ & $2(2,1)$ & 0,020 \\
\hline $2 / 3$ & $29(59,2)$ & $38(40)$ & \\
\hline $3 / 3$ & $17(34,7)$ & $55(57,9)$ & \\
\hline
\end{tabular}

Tabla 6. Distribución de la ingesta de la crema durante el cepillado según frecuencia de cepillado de pacientes que se les diligenció la ficha de notificación de fluorosis en las Clínicas Odontológicas Universidad Santo Tomás, 2014

\begin{tabular}{cccccc}
\hline \multicolumn{5}{c}{ Variables } & \multicolumn{5}{c}{ Frecuencia de cepillado con crema dental } \\
\hline $\mathrm{n}(\%)$ & Una vez & Dos veces & Tres veces & Cuatro veces & Valor p \\
& $28(19,4)$ & $75(52,1)$ & $38(26,4)$ & $3(2,1)$ & \\
\multicolumn{2}{r}{ Ingesta de crema dental durante el cepillado } & & & & \\
Sí & $7(12,7)$ & $32(58,2)$ & $14(25,5)$ & $2(3,6)$ & 0,280 \\
No & $21(23,6)$ & $43(48,3)$ & $24(27)$ & $1(1,1)$ & \\
\hline
\end{tabular}




\section{DISCUSIÓN}

En el presente estudio se llevó a cabo el diligenciamiento de la ficha de notificación obligatoria para los casos de fluorosis dental, en 144 niños que acudieron a consulta en las Clínicas Odontológicas de la Universidad Santo Tomás Campus Floridablanca en el II periodo del 2014, con el fin de realizar un análisis de los factores de mayor influencia que giran en torno a la alteración, tales como presencia de caries, estrato socioeconómico, aplicación de flúor, cantidad de crema dental utilizada, entre otros. Se logró identificar que hace falta educación y capacitación a los padres y cuidadores encargados de los niños en cuanto a la enseñanza y vigilancia de los hábitos adecuados de higiene; ya que se encontró que los factores asociados a fluorosis dental de mayor importancia son los relacionados con el uso de cremas dentales (cantidad entre $2 / 3$ y $3 / 3$ de la totalidad de la cabeza del cepillo, ingesta de crema dental durante el cepillado y responsable del cepillado). Hallazgos similares a los reportados en el 2010 por González, quienes en su trabajo percibieron hábitos poco favorables que pueden ser responsables del consumo de cantidades de flúor nocivas para la salud, tales como ingesta de crema durante el cepillado, proporción de pasta dental utilizada, donde lo normal fue que los padres manifestaran usar como parámetro entre la mitad del cepillo y el cepillo completo (17).

El estrato socioeconómico se presume que es otro factor de influencia, teniendo en cuenta que los participantes del presente estudio pertenecen en su mayoría a estratos 1,2 y 3 correspondientes en Colombia a nivel bajo-bajo, bajo y medio-bajo, respectivamente; lo cual está directamente relacionado con la menor disponibilidad de recursos y puede deberse, a su vez, a que hace falta mayor cobertura, por parte del Gobierno, a servicios de salud. Encontrándose relación con un trabajo realizado en Mérida, Venezuela en el 2008 en el que se concluyó que el riesgo relativo de padecer caries es de 2,21 cuando se es de pobreza relativa y que se podía demostrar la influencia que tiene el nivel socioeconómico sobre la prevalencia de caries dental (35).

De acuerdo con la clasificación de la lesión según Dean, los casos de fluorosis dental que más se encontraron en la Universidad Santo Tomás fueron leves, seguido de moderada y muy leve, hallazgos similares a los de otros estudios realizados en el país, tales como: 1998 en Medellín, Rionegro en 1999, Manizales en el 2000, Caldas 2005 y el estudio de Bermúdez en 2009 donde los mayores porcentajes de fluorosis según Dean se encontraban en los grados muy leve y leve seguidos de la forma moderada
(36). No obstante, que el grado de fluorosis no disminuya en el país puede ser producto de la exposición a múltiples fuentes de flúor sin control adecuado (consumo sal, cremas dentales), por lo que sería conveniente revisar los programas de salud bucal a nivel nacional e institucional, con el fin de identificar falencias y promover intensivamente cambios de hábitos para intentar disminuir la incidencia y severidad de fluorosis dental en Colombia.

Se sugiere anexar un ítem en la ficha de notificación obligatoria, en el que se registre el género y la edad, además del tipo de crema dental utilizada, lo que permita conocer la concentración en ppm de flúor en las cremas y así poder obtener datos más especíicos con los que se pueda realizar un mejor análisis de los casos de fluorosis dental. Además, es conveniente seguir apoyando e implementando las campañas de promoción y prevención dentro de las Clínicas Odontológicas a nivel institucional y departamental, con el fin de crear en el niño y adulto hábitos de higiene oral adecuados, y así lograr una mejor manipulación de las fuentes de exposición a flúor y alcanzar índices más favorables en salud bucal.

\section{Conclusiones}

Los factores relacionados con el uso inadecuado de cremas dentales siguen jugando un rol importante en la incidencia de fluorosis dental.

Hace falta educación en cuanto a los hábitos adecuados de higiene oral, como frecuencia de cepillado, cantidad de crema utilizada y acompañamiento oportuno en los primero años de vida del niño para evitar alteraciones como la fluorosis.

El nivel socioeconómico es un factor trascendental en cuanto a las condiciones de salud, bienestar integral de un individuo, en los hábitos saludables, costumbres de higiene y cuidado bucal.

\section{BIBLIOGRAFÍA}

1. Beltrán M. Investigar las consecuencias del efecto acumu- lativo del flúor, una necesidad imperante de la profesión odontológica. Rev. Colomb. Invest. Odontol. 2012;3(7):55-72.

2. Misnaza S. Protocolo de vigilancia y control centinela de la exposición a flúor - Instituto Nacional de Salud. Protocolos Sivigila [Internet] - 2011 dic [citado 2014 may 15], p. 01-46. Disponible en: http://www.ins.gov.co/lineas-deaccion/SubdireccionVigilancia/sivigila/Protocolos\%20SIVIGILA/EXPOSICION\%20FLUOR\%20(centinela).pdf.

3. González MA, Pazos GA. Prevalencia de Fluorosis en niños de 10 a 14 años en el municipio de Cajibio de marzo a mayo del 2011 [tesis]-Universidad Escuela Administración de Negocios. 2011.

4. Rivera S, Godorccci S, Borgel L, Díaz E, Fuchs T, Martin I. Flúor: potenciales efectos adversos. Rev. Chil. pediatr. 1993;64(4):278- 283. 
5. Loyola JP, Pozos A, Hernández JC, Hernández JF. Fluorosis en dentición temporal en un área con hidrofluorosis endémica. Salud pública Méx. 2000;42:194-200.

6. Instituto Nacional de Salud. Informe del evento vigilancia centinela de la exposición a flúor, hasta el periodo epidemiológico tres del año 2014. Bogotá, 2014 [Internet]. [citado 2014 sep 2]. Disponible en: http:// www.ins.gov. co/lineas-de-accion/SubdireccionVigilancia/ Informe $\% 20$ de\%20Evento\%20Epidemiolgico/EXPOSI- CION\%20A\%20 FLUOR \%20Periodo\%20III\%202014.pdf.

7. Azpeitia M, Rodríguez M, Sánchez MA. Prevalencia de fluorosis dental en escolares de 6 a 15 años de edad. Rev. Med. Inst. Mex. 2008;1(46):67-72.

8. García M, Sosa M, Cuéllar L, Rodríguez L, Cangas R. Sistema de vigilancia de fluoruro en aguas de consumo en Cuba. Rev. Cub. de higi. y epid. 2002;40(2):136-142.

9. Tovar S, Rengifo H, Cuéllar C, Rodríguez A, Jaramillo M, Peñalosa E, et al. IV Estudio Nacional de Salud Bucal ENSAB IV - Ministerio de Salud y Protección Social, Dirección de Promoción y Prevención, Subdirección Enfermedades no Transmisibles [Internet] - Bogotá, 23 Sept. 2013 Citado Feb. 2015, p. 1- 22. Disponible en: http://www. minsalud.gov.co.

10. Concha SC, Celedon Y, Vera W, Poveda E, Muñoz C, Vergel $\mathrm{T}$, et al. Prevalencia de fluorosis dental en escolares de 6 a 15 años de edad de la zona urbana de Bucaramanga. UstaSalud. 2003;(2):73-82.

11. Blanco H, Durán L, Neira LN, Pourgoshtasbi L, Carvajal LC, Concha SC. Comparación de los niveles de fluorosis dental en escolares de dos municipios de Santander. UstaSalud. 2008;(7):108-116.

12. Montaña MA. Guía de fluorosis dental. Normas técnicas de la fluorosis dental. Secretaría de Salud Departamental, Gobernación del Huila [Internet] - Dic 2008 - Citado Abril 2013 - p. 01-82 - Disponible en: file:///C:/Users/Disponible/Desktop/guia_fluorosis_dental_huila.pdf.

13. Arrieta K, González F, Pomares Cueto M. Conocimientos y prácticas sobre fluorosis dental en odontólogos y auxiliares hospital local Cartagena de Indias. Rev. Colomb. Inv. Odonto. 2012;3(7):1-9.

14. Decreto Número 3039 de 10 Agosto de 2007 - Diario Oficial 46716 de 10 Agosto de 2007.

15. Santana Y, Suárez I, Rincón MC, Morón A, García R. Prevalencia de fluorosis y caries dental en niños y adolescentes del municipio Baralt. Cienc Odon. 2012;9(1):7-16.

16. González F, Carmona L, Díaz A. Percepción de ingesta de flúor a través del cepillado dental en niños colombianos. Rev. Cubana Estomatol. 2010;3(47):1-5.

17. Resolución Número 3577 de 2006 - Dada en Bogotá, D.C, a los 28 días del mes de Septiembre del año 2006 - Diario Oficial No. 46.411 de 4 de octubre de 2006.

18. Arellano LA, Fleitas AT, Ramírez AC. Prevalencia e intensidad de fluorosis dental en escolares de 10-13 años de edad en San Carlos y Santa Bárbara del Zulia, Venezuela. Ac Odontol. Ven. [Internet] - 1998 [citado 2013 ago 2]. Disponible en: http://www.actaodontologica.com/ediciones/1998/2/prevalencia_intensidad_fluorosis_dental escolares.asp\#top.

19. Cárdenas D. Fundamentos de odontología - Odontología Pediátrica $3^{a}$ ed. Medellín Colombia.: Corporación para Investigaciones Biológicas, CIB. 2003.
20. Agudelo A, Martínez L, Madrid M, Vivares A, Rocha A. Panorama de la fluorosis dental en Colombia: una revisión exploratoria de la literatura. Rev. Cient. Ponti. Univ. Javeri. $2013 ; 1(32)$ :133-145.

21. Hurtado R, Gardea J. Estimación de la exposición a fluoruros en Los Altos de Jalisco, México. Salud Pública Méx. 2005;45(1):58-63.

22. Bordoni N, Escobar A, Castillo R. Odontología Pediátrica. La salud bucal del niño y el adolescente en el mundo actual, $1^{\mathrm{a}}$ ed. Buenos Aires: Editorial Panamericana. 2010.

23. DeLong L, Borkhart N. General and Oral Pathology for the Dental Hygienist, $2^{\mathrm{a}}$ Edition. Lippincott Williams \& Wilkins. 2013.

24. Saldarriaga A. Fluorosis dental y flúor sistémico en fundamentos de odontología pediátrica. Cárdenas Jaramillo D. [Editor] - 4a ed. Medellín, Colombia: Fondo Editorial CIB. 2009.

25. Gutiérrez SJ. (Editor). Fundamentos de ciencias básicas aplicadas a la odontología, $1^{\text {a }}$ ed. Bogotá: Editorial Pontificia Universidad Javeriana. 2006.

26. Slootweg P. Dental Pathology: A Practical Introduction, $2^{\mathrm{a}}$ ed. Springer Science \& Business Media. 2013.

27. Cameron AC, Widmer RP. [Editores]. Manual de Odontología Pediátrica. Madrid, España: Harcourt Brace de España S.A. 1998.

28. Decreto Número 547 de 1996, Santa Fe de Bogotá, D. C, el 19 de marzo de 1996. Diario Oficial No. 42.748, del 20 de marzo de 1996.

29. Decreto Número 1575 de 2007, Bogotá, D. C., a los 9 días del mes de mayo de 2007. Diario Oficial 46623 de mayo 09 de 2007.

30. Resolución Número 2115 de 2007. Bogotá, D. C, a los 22 días del mes de junio de 2007. Diario Oficial No. 46.679 de 04 de Julio de 2007.

31. Ley 35 de 1989, Bogotá, D.E, 9 de marzo de 1989. Diario Oficial No. 38.733.

32. Ley Estatutaria 1266 de 2008 . Fecha de entrada en vigencia 31 Diciembre de 2008. Diario Oficial 47.219 de Diciembre 31 de 2008.

33. Ley 1273 de 2009. Bogotá, D. C. a los 5 días del mes de enero de 2009. Diario Oficial No. 47.223 de 5 de enero de 2009.

34. Resolución No. 008430 de 1993. Santafé de Bogotá, D.C. el 04 de Octubre de 1993. Diario Oficial No. 46506.

35. Hernández M. Relación entre estrato socio-económico y la presencia de caries dental en primeros molares inferiores en alumnos de la U.E. Eloy Paredes y U.E La Salle Mérida-Venezuela 2008. Rev Academia. 2009;8(15):55-61.

36. Bermúdez P. Prevalencia de fluorosis dental en escolares de 6 a 12 años en la Institución Educativa Javiera Londo- El Bosque, Medellín, Colombia 2009. Rev.Colom. Inv. Odont. 2010;(1):1-7.

\section{Correo electrónico de los autores:}

Olga Viviana cañas: vivi_2709@hotmail.com Andrea carolina Velásquez: andreacarolina_1306@hotmail.com Geyme Paola zapata: gepazare@hoymail.com Francisco Tirado Santamaría: pachotirado@outlook.es 